\title{
Performance Analysis of Output Threshold-Based Incremental Multiple-Relay Combining Scheme with Adaptive Modulation for Cooperative Networks
}

\author{
Kyu-Sung Hwang ${ }^{1}{ }^{1}$ and MinChul Ju $\mathbb{D}^{2}$ \\ ${ }^{1}$ Department of Electronics Engineering, Kyungil University, Gyeongbuk, Republic of Korea \\ ${ }^{2}$ School of Electrical Engineering, Kookmin University, Seoul, Republic of Korea \\ Correspondence should be addressed to MinChul Ju; mcju@kookmin.ac.kr
}

Received 23 August 2017; Accepted 12 December 2017; Published 11 January 2018

Academic Editor: Nazrul Islam

Copyright ( $) 2018$ Kyu-Sung Hwang and MinChul Ju. This is an open access article distributed under the Creative Commons Attribution License, which permits unrestricted use, distribution, and reproduction in any medium, provided the original work is properly cited.

\begin{abstract}
In this paper, we propose an output threshold-based incremental multiple-relay combining scheme for cooperative amplify-andforward relay networks with nonidentically distributed relay channels. Specifically, in order to achieve the required performance, we consider both conventional incremental relaying and multiple-relay selection where relays are adaptively selected based on a predetermined output threshold. Moreover, the adaptive modulation technique is adopted by our proposed scheme for satisfying both the spectral efficiency and the required error rate. For the proposed scheme, we first derive an upper bound of the output combined signal-to-noise ratio and then provide its statistics such as cumulative distribution function (CDF), probability density function (PDF), and moment generating function (MGF) over independent, nonidentically distributed Rayleigh fading channels. Additionally, we analyze the system performance in terms of average spectral efficiency, average bit error rate, outage probability, and system complexity. Finally, numerical examples show that our proposed scheme leads to a certain performance improvement in the cooperative networks.
\end{abstract}

\section{Introduction}

In modern wireless communications, diversity techniques have received great attention to mitigate wireless channel impairments. Specifically, cooperative networks with the help of relays can be used to make broader coverage as well as to increase robustness against multipath fading [1]. However, cooperative networks lead to a loss in spectral efficiency because more resources (two frequency bands or two time slots) are used for half-duplex transmission [1]. To avoid such a loss in spectral efficiency by utilizing relays, incremental relaying scheme was proposed in [1] where a relay is used only if the direct transmission between the source and the destination does not support a predetermined performance. In other words, a relay is not used when the quality of the direct link exceeds a certain threshold, usually, in terms of signal-to-noise ratio (SNR).

There has been a lot of research studies on the cooperative network with multiple relays [2-7] because a spatial diversity gain can be achieved by utilizing multiple relays in a network like the antenna diversity system. However, utilizing multiple relays causes transmission efficiency losses as the number of relays increases. For examples, when $m$ relays are utilized for the cooperative communication, it is required to have $m+1$ system resources. In order to avoid such huge losses, relay selection has been researched in the literature [2] that can obtain the diversity order as much as the space time-coded multiple-relay scheme [3] whereas the system complexity can be reduced by a use of only two resources. More specifically, opportunistic relaying was proposed in [2] where the system used only the best one among relays based on the relay selection criteria and proved that the same diversity-multiplexing tradeoff could be achieved as the space time-coded cooperative network [3]. Authors in [4] provided the diversity order of single relay selection scheme and studied on multiple-relay selection schemes with the SNR-optimal/suboptimal criteria as well as their diversity orders. Authors in [5] proposed the multiple-relay selection 
scheme in large AF relay network based on exploiting the sparsity of the relay gain vector. Additionally, authors in [6] proposed an output threshold-based multiple-relay selection scheme in the amplify-and-forward (AF) transmission where $L_{c}$ relays are sequentially selected out of $L$ relays according to the predetermined threshold at the destination and gave performance analyses over the independent, identically distributed (i.i.d.) Rayleigh fading channels. Recently, the authors in [7] investigated an adaptive multiple-relay selection scheme in conjunction with the incremental relaying scheme and provided its link performance analyses over the independent but nonidentically distributed (i.ni.d.) Rayleigh fading channels, where the usage of relays (one or multiple) is decided based on the traditional incremental relaying fashion [3].

Moreover, in a time-varying channel, adaptive transmission where the transmission rate is controlled according to the channel state information (CSI) is a well-known technique for increasing spectral efficiency while satisfying the required error performance [8]. Specifically, in order to increase spectral efficiency, cooperative diversity systems with variable rate transmission have been widely researched in the literature [9-13]. For examples, Hwang et al. in [9] proposed the incremental opportunistic relaying with adaptive modulation (IOR-AM) scheme, combined the incremental relaying and best-relay selection schemes, and showed a certain improvement in spectral efficiency based on adaptive $M$-ary quadrature amplitude modulation $(M$ QAM). In [10], authors studied the performance analysis of adaptive transmission of multiple-relay AF cooperative system without relay selection. In [11], authors summarized the work done on relay selection in AF cooperative systems with fixed-rate transmission as well as with discrete-rate adaptive transmission. B. Lee and C. Lee in [12] considered the incremental hop selection with adaptive modulation in multiple relays network. Sun et al. in [13] analyzed the twoway AF relay network with adaptive modulation and coding.

1.1. Motivation and Our Contribution. Existing relay selection schemes [2, 4, 5, 9] unfortunately require complete CSI of all relay paths at every transmission for relay ordering, which can lead system overhead excessive in high speed wireless communications. In detail, a complexity of the best-relay selection schemes increases exponentially with the number of relays. Though the previous best-relay selection scheme, IOR-AM in [9], can achieve better spectral efficiency than multiple-relay selection schemes, its error rate and outages can be worse and the system complexity by estimations of relay channels can be larger. Therefore, a new multiplerelay selection scheme that offers a better tradeoff in spectral efficiency, error performance, and system complexity is desirable. Motivated by this, we propose output thresholdbased incremental multiple-relay combining with adaptive modulation (IMRC-AM) that is an extended work of the conventional incremental relaying in a distributed multiplerelay network and that can achieve both high spectral efficiency and high diversity gain. Instead just checking some fixed values of output thresholds at the destination like the conventional diversity system [14] and relaying systems $[1,6]$, we use the minimum threshold of adaptive modulation as the output threshold in order to achieve both spectral efficiency and error performance at a given channel condition where the minimum threshold of adaptive modulation is variable according to error performance requirements.

In a view of system complexity, we estimate and select only $L_{c}$ relays out of $L$ relays in consecutive order where $L_{c}$ is from 0 to $L\left(0 \leq L_{c} \leq L\right)$ in our proposed IMRCAM. For instance, no relay is used if the direct path can realize the desired performance (i.e., $L_{c}=0$ ), whereas only $L_{c}$ relays are utilized to obtain the target performance according to channel conditions when the direct path fails to support the predetermined performance. Therefore, our proposed scheme can offer a reduced complexity compared to the best-relay selection scheme (i.e., IOR-AM [9]) while the error requirements are satisfied, because it does not require the ordering of relays by checking all channel paths.

For the performance analyses of the proposed IMRC-AM, we consider AF transmission at the relay and the conventional upper bound of the output SNR at the destination. Given this upper bound of the output SNR in AF transmission [15], some statistics such as the cumulative distribution function (CDF), probability density function (PDF), and moment generating function (MGF) are evaluated in a closed-form expression over the independent but nonidentically distributed (i.ni.d.) Rayleigh fading relay channels. By using the provided statistics of the proposed IMRC-AM, we analyze the performances of the proposed system such as average spectral efficiency, average bit error rate (BER), outage probability, and system complexity. Finally, we give some numerical examples to show the system efficiency and to verify our analytical results.

\section{System Description}

We consider an AF cooperative system where one source node, one destination node, and $L$ relay nodes are utilized with a single antenna, and there exist feedback channels in the source-destination link and the relays-destination links. As stated in Section 1, the proposed system model is based on incremental relaying [1] where the source-destination transmission is successful when its channel is good enough to support a certain performance and the cooperative transmission operates in a half-duplex mode requiring two time slots when some relays help a transmission. At the first time slot, the source broadcasts its signal to the destination as well as the relays. When the direct link fails to support a certain threshold, that is, minimum threshold of adaptive modulation, the selected relays transmit their amplified signals to the destination via orthogonal channeling (between the relays and the destination) at the second time slot. In addition, we consider an AF relaying system with the powerscaled gain at $i$ th relay node $G=1 / \sqrt{\mathrm{E}\left[\left|r_{i}\right|^{2}\right]}$, and its received SNR at the destination via $i$ th relay can be expressed as $\gamma_{\mathrm{AF}}^{i}=$ $\gamma_{s, i} \gamma_{i, d} /\left(\gamma_{s, i}+\gamma_{i, d}+1\right)$, where $r_{i}$ is the received signal at $i$ th relay and $\gamma_{s, i}$ and $\gamma_{i, d}$ are instantaneous SNRs of channels source/ith relay and $i$ th relay/destination, respectively.

In our proposed IMRC-AM, it is assumed that there is a certain sequential order to estimate relay channel paths 
and the receiver of the destination combines those paths in consecutive order based on the output threshold at the destination. In this work, the destination decides the number of the operated relays according to channel conditions; thus it is assumed that the destination knows the sequential order in successively selecting relay nodes in an incremental manner and performs relay selection via orthogonal feedback channels from relays during a guard period of the second time slot. From the concepts of incremental relaying and adaptive modulation, only the direct path between the source and destination nodes is used if the SNR of the direct path exceeds the minimum threshold of adaptive modulation, $\gamma_{T}^{1}$. If the direct path fails to support the minimum threshold, the receiver at the destination adaptively and successively combines as many relay paths as necessary in order to have the desired output SNR exceeding the minimum threshold. From the above step, let $L_{c}$ denote the number of the combined relay paths, where $0 \leq L_{c} \leq L$. Once the output combined SNR $\gamma_{c}$ with $L_{c}$ relays satisfies the minimum threshold, the destination decides the constellation size according to the switching threshold of adaptive $M$-QAM, where $\gamma_{T}^{n}$ denotes the threshold that the $n$ bits can be transmitted while satisfying the certain error performance. The detailed mode of operation is described below:

(1) Only the direct path between the source and the destination is used when its received SNR, $\gamma_{s, d}$, is higher than the minimum threshold SNR of adaptive modulation, $\gamma_{T}^{1}$. (i.e., $\gamma_{s, d} \geq \gamma_{T}^{1}$ and $L_{c}=0$ ).

(2) The destination decides a constellation size based on the output SNR and then informs the source via a feedback channel.

(3) If $\gamma_{s, d}$ is below $\gamma_{T}^{1}$, a cooperative transmission operates during the second time slot. The destination wakes the first relay up and with a request to send its pilot signal.

(4) The destination starts estimating the first relay path channel and then calculates the combined SNR (i.e., $\left.\gamma_{c}=\gamma_{s, d}+\gamma_{\mathrm{AF}}^{1}\right)$. If $\gamma_{c} \geq \gamma_{T}^{1}$, only the first relay path is used and the destination decides a constellation size based on the combined SNR. If $\gamma_{c} \leq \gamma_{T}^{1}$, the destination continues to estimate the next relay path channel and then calculates the combined SNR (i.e., $\left.\gamma_{c}=\gamma_{s, d}+\gamma_{\mathrm{AF}}^{1}+\gamma_{\mathrm{AF}}^{2}\right)$.

(5) In the same manner, the first $L_{c}$ relay paths $\left(1 \leq L_{c} \leq\right.$ $L-1)$ are combined where the output combined SNR of the direct path and first $L_{c}$ relay paths is above the minimum threshold SNR (i.e., $\gamma_{c} \geq \gamma_{T}^{1}$ ).

(6) All the $L$ relay paths are used when the output combined SNR with the first $L-1$ relay paths does not achieve the minimum threshold (i.e., when $L_{c}=L-1$, $\left.\gamma_{c} \leq \gamma_{T}^{1}\right)$. A constellation size is decided based on the output combined SNR with $L$ relay paths, and the outage event occurs when it is still less than the minimum threshold.

Based on the mode of operation, we can observe the probability of the output combined $\operatorname{SNR} \gamma_{c}$, mathematically, as

$$
\begin{aligned}
& \operatorname{Pr}\left[\gamma_{c}=\gamma_{s, d}+\sum_{i=0}^{L_{c}} \gamma_{\mathrm{AF}}^{i}\right] \\
& = \begin{cases}\operatorname{Pr}\left[\gamma_{s, d} \geq \gamma_{T}^{1}\right], & L_{c}=0 \\
\operatorname{Pr}\left[\gamma_{s, d}+\gamma_{\mathrm{AF}}^{1} \geq \gamma_{T}^{1}, \gamma_{s, d}<\gamma_{T}^{1}\right], & L_{c}=1 \\
\operatorname{Pr}\left[\gamma_{s, d}+\sum_{i=1}^{L_{c}} \gamma_{\mathrm{AF}}^{i} \geq \gamma_{T}^{1}, \gamma_{s, d}+\sum_{i=1}^{L_{c}-1} \gamma_{\mathrm{AF}}^{i}<\gamma_{T}^{1}\right], & 2 \leq L_{c} \leq L-1 \\
\operatorname{Pr}\left[\gamma_{s, d}+\sum_{i=1}^{L-1} \gamma_{\mathrm{AF}}^{i}<\gamma_{T}^{1}\right], & L_{c}=L .\end{cases}
\end{aligned}
$$

Since the event that $L_{c}$ relay paths are selected is mutually exclusive, we can write the CDF of $\gamma_{c}$ by the total probability theorem as

$$
\begin{aligned}
P_{\gamma_{c}}(x)= & \operatorname{Pr}\left[\gamma_{T}^{1} \leq \gamma_{s, d}<x\right]+\operatorname{Pr}\left[\gamma_{s, d}<\gamma_{T}^{1} \leq \Gamma_{1}<x\right] \\
& +\sum_{l=2}^{L-1} \operatorname{Pr}\left[\Gamma_{l-1}<\gamma_{T}^{1} \leq \Gamma_{l}<x\right] \\
& +\operatorname{Pr}\left[\Gamma_{L-1}<\gamma_{T}^{1}, \Gamma_{L}<x\right]
\end{aligned}
$$

where $\Gamma_{l}=\gamma_{s, d}+\sum_{i=1}^{l} \gamma_{\mathrm{AF}}^{i}$. Using (1) and (2), we can rewrite the CDF of $\gamma_{c}$ as

$$
\begin{aligned}
& P_{\gamma_{c}}(x) \\
& =\left\{\begin{array}{l}
P_{\gamma_{s, d}}(x)-P_{\gamma_{s, d}}\left(\gamma_{T}^{1}\right)+\int_{0}^{\gamma_{T}^{1}} \int_{\gamma_{T}^{1}-z}^{x-z} p_{\gamma_{s, d}}(z) p_{\gamma_{\mathrm{AF}}^{1}}(y) d y d z+\sum_{l=2}^{L-1} \int_{0}^{\gamma_{T}^{1}} \int_{\gamma_{T}^{1}-z}^{x-z} p_{\Gamma_{l-1}}(z) p_{\gamma_{\mathrm{AF}}^{l}}(y) d y d z+\int_{0}^{\gamma_{T}^{1}} \int_{0}^{x-z} p_{\Gamma_{L-1}}(z) p_{\gamma_{\mathrm{AF}}^{L}}(y) d y d z, \quad \gamma_{T}^{1}<x \\
P_{\Gamma_{L}}(x),
\end{array} \quad \gamma_{T}^{1} \geq x .\right.
\end{aligned}
$$

The corresponding PDF of $\gamma_{c}$ can be obtained by differentiating (3) with respect to $x$ as

$$
p_{\gamma_{c}}(x)= \begin{cases}p_{\gamma_{s d}}(x)+\int_{0}^{\gamma_{T}^{1}} p_{\gamma_{s d}}(z) p_{\gamma_{\mathrm{AF}}^{1}}(x-z) d z+\sum_{l=2}^{L} \int_{0}^{\gamma_{T}^{1}} p_{\Gamma_{l-1}}(z) p_{\gamma_{\mathrm{AF}}^{l}}(x-z) d z, & \gamma_{T}^{1}<x \\ p_{\Gamma_{L}}(x), & \gamma_{T}^{1} \geq x .\end{cases}
$$




\section{Performance Analysis over i.ni.d. Rayleigh Fading Channels}

3.1. Statistical Analysis of Output Combined SNR. In this section, we consider the upper bound of the output combined SNR $\gamma_{c}^{\text {ub }}$ since the statistics of $\gamma_{c}$ are not tractable in a closed-form expression. With the traditional maximal ratio combiner at the destination, the output combined SNR with $l$ relay paths, $\Gamma_{l}$, can be approximated by its upper bound as $\Gamma_{l}^{\mathrm{ub}}=\gamma_{s, d}+\sum_{i=1}^{l} \gamma_{\mathrm{AF}}^{\mathrm{ub}_{i}}=\gamma_{s, d}+\sum_{i=1}^{l} \min \left(\gamma_{s, i}, \gamma_{i, d}\right)$ [10]. Over the i.ni.d. Rayleigh fading channels, the CDF and PDF of $\Gamma_{l}^{\mathrm{ub}}$ are given by [10]:

$$
P_{\Gamma_{l}^{\mathrm{ub}}}(x)=1-\left[\widehat{\beta}_{0, l} e^{-x / \tau_{0}}+\sum_{i=1}^{l} \widehat{\beta}_{i, l} e^{-x / \tau_{i}}\right],
$$

$$
p_{\Gamma_{l}^{\mathrm{ub}}}(x)=\frac{\widehat{\beta}_{0, l}}{\tau_{0}} e^{-x / \tau_{0}}+\sum_{i=1}^{l} \frac{\widehat{\beta}_{i, l}}{\tau_{i}} e^{-x / \tau_{i}}
$$

respectively, where $\tau_{0}=\bar{\gamma}_{s, d}, \tau_{i}=\left(\bar{\gamma}_{s, i} \bar{\gamma}_{i, d}\right) /\left(\bar{\gamma}_{s, i}+\bar{\gamma}_{i, d}\right), \widehat{\beta}_{0,0}=$ $1, \widehat{\beta}_{0, l}=\prod_{i=1}^{l}\left(1-\tau_{i} / \tau_{0}\right)^{-1}, \widehat{\beta}_{i, l}=\left(1-\tau_{0} / \tau_{i}\right)^{-1} \prod_{k=1, k \neq i}^{l}(1-$ $\left.\tau_{k} / \tau_{i}\right)^{-1}$. In addition, the PDF of the upper bound of $\gamma_{\mathrm{AF}}^{l}$ is simply given as

$$
p_{\gamma_{\mathrm{AF}}^{\mathrm{ub}}}(x)=\frac{1}{\tau_{l}} e^{-x / \tau_{l}}
$$

The CDF and PDF of the upper bound of the output combined SNR $\gamma_{c}^{\text {ub }}$ over the i.ni.d. Rayleigh channels can be obtained by substituting (5) and (6) into (3) and (4) as

$$
\begin{aligned}
P_{\gamma_{c}^{\mathrm{ub}}}(x) & = \begin{cases}\sum_{l=0}^{L-1 l-1} \sum_{i=0}^{L} \lambda_{i, l}\left(e^{-\gamma_{T} / \tau_{l}}-e^{-x / \tau_{l}}\right)+\sum_{i=0}^{L-1}\left[\widehat{\beta}_{i, L-1}\left(1-e^{\gamma_{T} / \tau_{i}}\right)-\lambda_{i, L} e^{-x / \tau_{L}}\right], & \gamma_{T}<x \\
1-\sum_{l=0}^{L} \widehat{\beta}_{l, L} e^{-x / \tau_{l}}, & \gamma_{T} \geq x\end{cases} \\
p_{\gamma_{c}^{\mathrm{ub}}}(x) & = \begin{cases}\sum_{l=0}^{L} \sum_{i=0}^{L-1} \frac{\lambda_{i, l}}{\tau_{l}} e^{-x / \tau_{l}}, & \gamma_{T}<x \\
\sum_{l=0}^{L} \frac{\hat{\beta}_{l, L}}{\tau_{l}} e^{-x / \tau_{l}}, & \gamma_{T} \geq x,\end{cases}
\end{aligned}
$$

respectively, where $\lambda_{0,0}=1, \lambda_{i, j}=\left(\widehat{\beta}_{i, j-1} \tau_{j} /\left(\tau_{j}-\tau_{i}\right)\right)(1-$ $\left.e^{-\left(\left(\tau_{j}-\tau_{i}\right) / \tau_{i} \tau_{j}\right) \gamma_{T}}\right)$. Correspondingly, the MGF of $\gamma_{c}^{\mathrm{ub}}$ for the i.ni.d. Rayleigh fading channels can be given by

$$
\begin{aligned}
\mathscr{M}_{\gamma_{c}^{\mathrm{ub}}}(x)= & \sum_{l=0}^{L} \sum_{i=0}^{l-1} \lambda_{i, l}\left(\frac{1-e^{-\gamma_{T}\left(1 / \tau_{l}+s\right)}}{1+\tau_{l} s}\right) \\
& +\sum_{l=0}^{L} \widehat{\beta}_{l, L}\left(\frac{e^{-\gamma_{T}\left(1 / \tau_{l}+s\right)}}{1+\tau_{l} s}\right) .
\end{aligned}
$$

3.2. Average Probability of Error. There are performance criteria of interest, the probability of error defined relative to either symbol or bit errors [16]. Since we have obtained the MGF of $\gamma_{c}^{\text {ub }}$ over the i.ni.d. Rayleigh fading channels in (10), the lower bound of the average symbol error rate with the proposed scheme for different coherent modulations can be evaluated by the MGF-based approach [17]. For $M$-ary phase shift keying (MPSK) modulation, the average symbol error rate $P_{s}(E)$ can be calculated as $P_{s}(E)=(1 / \pi) \int_{0}^{(M-1) \pi / M} \mathscr{M}_{\gamma_{c}^{\mathrm{ub}}}\left(-\sin ^{2}(\pi / M) / \sin ^{2} \phi\right) d \phi$. In addition, the average bit error rate with the proposed scheme can be evaluated by averaging the conditional error rate over the PDF of the combined output SNR $p_{\gamma_{c}}(x)$ as $P_{b}(E)=$ $\int_{0}^{\infty} P_{b}(E \mid x) p_{\gamma_{c}}(x) d x$ where $P_{b}(E \mid x)$ is the conditional BER of an interested modulation and can be expressed in an approximation form for coherent modulations as $P_{b}(E \mid x) \approx$ $a Q(\sqrt{b x}) \cdot Q(\cdot)$ is the Gaussian $Q$-function defined as $Q(x)=$ $(1 / \sqrt{2 \pi}) \int_{x}^{\infty} e^{-t^{2} / 2} d t$. The parameters $a$ and $b$ depend on the type of approximation and the modulation type [16]. Thus, given the upper bound of the combined output SNR $\gamma_{c}^{\mathrm{ub}}$, the lower bound of average BER with the proposed scheme can be given by some manipulations as [18]

$$
\begin{aligned}
P_{b}(E)= & \int_{0}^{\gamma_{T}} \sum_{l=0}^{L} \sum_{i=0}^{l-1} \frac{a \lambda_{\mathrm{i}, l}}{\tau_{l}} \frac{\mathscr{Q}(\sqrt{b x})}{e^{x / \tau_{l}}} d x+\int_{\gamma_{T}}^{\infty} \sum_{l=0}^{L} \frac{a \widehat{\beta}_{l, L}}{\tau_{l}} \frac{\mathscr{Q}(\sqrt{b x})}{e^{x / \tau_{l}}} d x \\
= & \sum_{l=0}^{L} \sum_{i=0}^{l-1} a \lambda_{i, l}\left\{Q\left(\sqrt{b \gamma_{T}}\right)\left(1-e^{-\gamma_{T} / \tau_{l}}\right)-\frac{1}{2 \sqrt{\pi}}\left[\Gamma\left(\frac{1}{2}, \frac{b}{2} \gamma_{T}\right)-\sqrt{\pi}-\sqrt{\frac{b \tau_{l}}{b \tau_{l}+2}}\left(\Gamma\left(\frac{1}{2}, \frac{b \tau_{l}+2}{2 \tau_{l}} \gamma_{T}\right)-\sqrt{\pi}\right)\right]\right\} \\
& -\sum_{l=0}^{L} a \widehat{\beta}_{l, L}\left\{Q\left(\sqrt{b \gamma_{T}}\right)\left(1-e^{-\gamma_{T} / \tau_{l}}\right)+\frac{1}{2 \sqrt{\pi}}\left[\Gamma\left(\frac{1}{2}, \frac{b}{2} \gamma_{T}\right)+\sqrt{\frac{b \tau_{l}}{b \tau_{l}+2}} \Gamma\left(\frac{1}{2}, \frac{b \tau_{l}+2}{2 \tau_{l}} \gamma_{T}\right)\right]\right\},
\end{aligned}
$$


where $\Gamma(\alpha, x)$ is the incomplete gamma function defined as $\Gamma(\alpha, x)=\int_{x}^{\infty} e^{-t} t^{\alpha-1} d t$.

\section{Performance Analysis of the Proposed System with Adaptive Modulation}

4.1. Average Spectral Efficiency. Since we investigate the statistical analysis for an upper bound of the output combined SNR over Rayleigh fading channels in Section 3, we can obtain an upper bound for an average spectral efficiency of our proposed system as

$$
\eta_{\mathrm{IMRC}-\mathrm{AM}} \leq \sum_{n=1}^{N} n\left(d(n)+\sum_{l=1}^{L} \frac{c(n, l)}{l+1}\right)
$$

where $N$ is the maximum constellation size for adaptive modulation and $d(n)$ and $c(n, l)$ are the probabilities that each instantaneous SNR of the direct path and the combined path with the first $l$ relays falls in the $n$th region, respectively. The $1 /(l+1)$ in the second term of the parenthesis in the summation comes from the fact that the transmission with $l$ relays takes place in $l+1$ orthogonal channels or time slots. Moreover, in (12), the probability $d(n)$ can be obtained as $\int_{\gamma_{T}^{n}}^{\gamma_{T}^{n+1}} p_{\gamma_{s, d}}(x) d x$ for $n=1,2, \ldots, N$, and the probability $c(n, l)$ for $n=1,2, \ldots, N$, can be given by

$$
c(n, l)=\left\{\begin{array}{l}
\int_{0}^{\gamma_{T}^{1}} \int_{\gamma_{T}^{1}-z}^{\gamma_{T}^{n+1}-z} p_{\gamma_{s, d}}(z) p_{\gamma_{\mathrm{AF}}}^{\mathrm{ub}}(y) d y d z-\int_{0}^{\gamma_{T}^{1}} \int_{\gamma_{T}^{1}-z}^{\gamma_{T}^{n}-z} p_{\gamma_{s, d}}(z) p_{\gamma_{\mathrm{AF}}^{\mathrm{ub}} l}(y) d y d z, \quad l=1 \\
\int_{0}^{\gamma_{T}^{1}} \int_{\gamma_{T}^{1}-z}^{\gamma_{T}^{n+1}-z} p_{\Gamma_{l-1}^{\mathrm{ub}}}(z) p_{\gamma_{\mathrm{AF}}^{\mathrm{ub}}}(y) d y d z-\int_{0}^{\gamma_{T}^{1}} \int_{\gamma_{T}^{1}-z}^{\gamma_{T}^{n}-z} p_{\Gamma_{l-1}^{\mathrm{ub}}}(z) p_{\gamma_{\mathrm{AF}}^{\mathrm{ub} l_{l}}}(y) d y d z, \quad l=2, \ldots, L-1 \\
\int_{0}^{\gamma_{T}^{1}} \int_{0}^{\gamma_{T}^{n+1}-z} p_{\Gamma_{l-1}^{\mathrm{ub}}}(z) p_{\gamma_{\mathrm{AF}}^{\mathrm{ub}} l}(y) d y d z-\int_{0}^{\gamma_{T}^{1}} \int_{0}^{\gamma_{T}^{n}-z} p_{\Gamma_{l-1}^{\mathrm{ub}}}(z) p_{\gamma_{\mathrm{AF}}^{\mathrm{ub}}}(y) d y d z, \quad l=L .
\end{array}\right.
$$

Note that $\gamma_{T}^{N+1}=\infty$. For the i.ni.d. Rayleigh fading channels, $d(n)$ and $c(n, l)$ can be given by

$$
\begin{aligned}
d(n) & =e^{-\gamma_{T}^{n} / \gamma_{s, d}}-e^{-\gamma_{T}^{n+1} / \gamma_{s, d},} \\
c(n, l) & = \begin{cases}\lambda_{0,1}\left(e^{-\gamma_{T}^{n} / \tau_{1}}-e^{-\gamma_{T}^{n+1} / \tau_{1}}\right), & l=1 \\
\sum_{i=0}^{l-1} \frac{\hat{\beta}_{i, l-1} \tau_{l}}{\tau_{l}-\tau_{i}}\left(1-e^{-\left(\left(\tau_{l}-\tau_{i}\right) / \tau_{i} \tau_{l}\right) \gamma_{T}^{1}}\right) \times\left(e^{-\gamma_{T}^{n} / \tau_{l}}-e^{-\gamma_{T}^{n+1} / \tau_{l}}\right), & l=2, \ldots, L\end{cases}
\end{aligned}
$$

respectively.

4.2. Average Bit Error Rate. For coherent $M$-QAM with twodimensional Gray coding, the approximate BER expressions are given by [16, Eq. 9.31]

$$
\operatorname{BER}(n, \gamma) \approx \begin{cases}Q(\sqrt{2 \gamma}), & n=1 \\ Q(\sqrt{\gamma}), & n=2 \\ \frac{4}{n} Q\left(\sqrt{\frac{3 \gamma}{2^{n}-1}}\right), & n \geq 3,\end{cases}
$$

where $n=\log _{2} M$. The switching thresholds for $n$ bits transmission $\gamma_{T}^{n}$, in which the system will operate at a BER below the required target, can be chosen according to (16). For the given discrete-rate adaptive $M$-QAM, the average BER (ABER) of the proposed scheme can be approximated by the ratio of the average number of bits in error over the average number of transmitted bits as

$$
\mathrm{ABER} \simeq \frac{1}{\eta_{\mathrm{IMRC}-\mathrm{AM}}} \sum_{n=1}^{N} n\left(\overline{\mathrm{BER}}_{d(n)}+\sum_{l=1}^{L} \frac{\overline{\mathrm{BER}}_{c(n, l)}}{l+1}\right),
$$

where

$$
\begin{aligned}
\overline{\operatorname{BER}}_{d(n)} & =\int_{\gamma_{T}^{n}}^{\gamma_{T}^{n+1}} \operatorname{BER}(n, x) p_{\gamma_{s, d}}(x) d x, \\
\overline{\operatorname{BER}}_{c(n, l)} & =\int_{\gamma_{T}^{n}}^{\gamma_{T}^{n+1}} \operatorname{BER}(n, x) p_{\gamma_{c(l)}^{\mathrm{ub}}}(x) d x,
\end{aligned}
$$

where $p_{\gamma_{s, d}}(x)$ and $p_{\gamma_{c(l)}^{\mathrm{ub}}}(x)$ over the i.ni.d Rayleigh fading channels are given by $p_{\gamma_{s, d}}(x)=e^{-x / \bar{\gamma}_{s, d}} / \bar{\gamma}_{s, d}$ and $p_{\gamma_{c(l)}^{\text {ub }}}(x)=$ $\sum_{i=0}^{l} \sum_{j=0}^{i-1}\left(\lambda_{j, i} / \tau_{i}\right) e^{-x / \tau_{i}}$, respectively. In addition, (18) can be evaluated by taking similar manipulations with (11). For instance, $\overline{\operatorname{BER}}_{c(n, l)}$ can be obtained as 


$$
\begin{aligned}
& \overline{\operatorname{BER}}_{c(n, l)}=\int_{\gamma_{T}^{n}}^{\gamma_{T}^{n+1}} \sum_{i=0}^{l} \sum_{j=0}^{i-1} \frac{a \lambda_{j, i}}{\tau_{i}} Q(\sqrt{b x}) e^{-x / \tau_{i}} d x=\sum_{i=0}^{l} \sum_{j=0}^{i-1} a \lambda_{j, i}\left\{Q\left(\sqrt{b \gamma_{T}^{n+1}}\right)\left(1-e^{-\gamma_{T}^{n+1} / \tau_{i}}\right)-Q\left(\sqrt{b \gamma_{T}^{n}}\right)\left(1-e^{-\gamma_{T}^{n} / \tau_{i}}\right)\right. \\
& \left.-\frac{1}{2 \sqrt{\pi}}\left[\Gamma\left(\frac{1}{2}, \frac{b}{2} \gamma_{T}^{n+1}\right)-\Gamma\left(\frac{1}{2}, \frac{b}{2} \gamma_{T}^{n}\right)-\sqrt{\frac{b \tau_{i}}{b \tau_{i}+2}}\left(\Gamma\left(\frac{1}{2}, \frac{b \tau_{i}+2}{2 \tau_{i}} \gamma_{T}^{n+1}\right)-\Gamma\left(\frac{1}{2}, \frac{b \tau_{i}+2}{2 \tau_{i}} \gamma_{T}^{n}\right)\right)\right]\right\},
\end{aligned}
$$

where $a=4 / n$ and $b=3 /\left(2^{n}-1\right)$.

4.3. Outage Probability. For the proposed system with adaptive modulation, the outage event can happen when the system cannot transmit any bits according to adaptive modulation even it uses full system resources and, equivalently, when the combined output SNR with $L$ relay paths is smaller than the minimum threshold (i.e., $\Gamma_{L}<\gamma_{T}^{1}$ ). Therefore, we can evaluate the lower bound for the outage probability of the proposed system as $P_{\text {out }}^{\mathrm{lb}}=P_{\Gamma_{L}^{\mathrm{lb}}}\left(\gamma_{T}^{1}\right)$.

4.4. System Complexity. In this subsection, we focus on how many relays are estimated for the multiple-relay selection in the proposed incremental relay combining fashion. In our scenario, the $l$ th relay will be added when the current combined output SNR with $l-1$ relays is below the minimum threshold $\gamma_{T}^{1}$. Hence, the probability that the $l$ th relay is used can be given as

$$
\begin{aligned}
& \operatorname{Pr}\left[L_{c}=l\right] \\
& = \begin{cases}1-P_{\gamma_{s, d}}\left(\gamma_{T}^{1}\right), & l=0 \\
\int_{0}^{\gamma_{T}^{1}} \int_{\gamma_{T}^{1}-y}^{\infty} p_{\gamma_{s, d}}(y) p_{\gamma_{\mathrm{AF}}^{1}}(x) d x d y, & l=1 \\
\sum_{k=2}^{l} \int_{0}^{\gamma_{T}^{1}} \int_{\gamma_{T}-y}^{\infty} p_{\Gamma_{k-1}}(y) p_{\gamma_{\mathrm{AF}}^{k}}(x) d x d y, & 2 \leq l \leq L-1 \\
P_{\Gamma_{L-1}}\left(\gamma_{T}^{1}\right), & l=L .\end{cases}
\end{aligned}
$$

Since we know the statistics of $\Gamma_{i}^{\mathrm{ub}}$ and $\gamma_{\mathrm{AF}}^{\mathrm{ub}_{j}}$ instead of $\Gamma_{i}$ and $\gamma_{\mathrm{AF}}^{j}$ in (20), finally, the lower bound of the average number of estimated relays with the proposed scheme can be calculated by substituting (5), (6), and (7) into (20) as

$$
\begin{aligned}
N_{A}^{\mathrm{lb}}= & L-L \sum_{i=0}^{L-1} \widehat{\beta}_{i, L-1} e^{-\gamma_{T}^{1} / \tau_{i}} \\
& +\sum_{l=1}^{L-1} l\left[\sum_{j=1}^{l} \sum_{k=0}^{j-1} \frac{\widehat{\beta}_{k, j-1} \tau_{j}}{\tau_{k}-\tau_{j}} \times\left(e^{-\gamma_{T}^{1} / \tau_{k}}-e^{-\gamma_{T}^{1} / \tau_{j}}\right)\right] .
\end{aligned}
$$

\section{Numerical Examples}

For the numerical examples, we consider the i.ni.d. Rayleigh fading channels per hop where the average SNR are set as $\bar{\gamma}=\bar{\gamma}_{s, d}, \bar{\gamma}_{s, i}=d_{s, r_{i}}^{-\alpha} \bar{\gamma}$, and $\bar{\gamma}_{i, d}=d_{r_{i}, d}^{-\alpha} \bar{\gamma}$ for $i=1, \ldots, L$. In detail, we consider a radio propagation channel model [19] which is referred to as a path-loss model over distance where all the channels are normalized by the distance between the source and the destination set as $d_{s, i}+d_{i, d}=d_{s, d}$. Specifically, we set the path-loss exponent and the normalized distance between the source and the relays as $\alpha=3$ and $d_{s, r_{i}}=0.5+0.1 i$, respectively. In addition, the maximum constellation size $N$ is 8 and the target BER for adaptive modulation is $10^{-6}$ for Figures 1-4.

In Figure 1, we compare the average spectral efficiency of the proposed IMRC-AM with the IOR-AM [9], the conventional incremental relaying with adaptive modulation (IR$\mathrm{AM}$ ), and the noncooperative transmission. In the examples of comparison, the IOR-AM [9] uses only the best relay among relay candidates and the IR-AM has one relay in a cooperative network. In addition, we draw the performance bound for the average spectral efficiency of the IMRC-AM to verify our analytical result. Interestingly, the performance improvement by the IMRC-AM is observed in the low SNR regions in comparison with the IOR-AM [9] since the outage corresponding to event that the IOR-AM scheme cannot support the minimum threshold usually occurs in the low SNR region. This is an example that the proposed scheme can offer a better tradeoff between the spectral efficiency and the error performance. From a practical point of view, the IOR-AM scheme requires the estimation of all channels of relay paths when the cooperation is performed, whereas the proposed IMRC-AM scheme incrementally estimates the relay paths. Thus, as shown in Figure 2, the system complexity of the proposed scheme in terms of the average number of estimated relays is much lower than the previous IOR$\mathrm{AM}$ in overall SNR regions even it offers better average spectral efficiency performance in some low SNR regions. For example, in Figures 1 and 2, from 0 to $5 \mathrm{~dB}$ in the average $\mathrm{SNR}$, the proposed scheme achieves better performance in the average spectral efficiency while requiring lower system complexity. Additionally, the performance analyses of lower bound on the average number of estimated relays are also well matched to the simulation results.

The numerical examples in Figure 3 show the proposed scheme can satisfy the required BER performance. In addition, our analytical results are in good agreement with our simulation results.

In Figure 4, we illustrate the outage probability of the proposed IMRC-AM in comparison with IOR-AM, IR-AM, and noncooperation. In this example, we can observe that a significant improvement of the outage performance can be obtained. This is because the proposed IMRC-AM focuses on increasing the probability of a transmission within given 


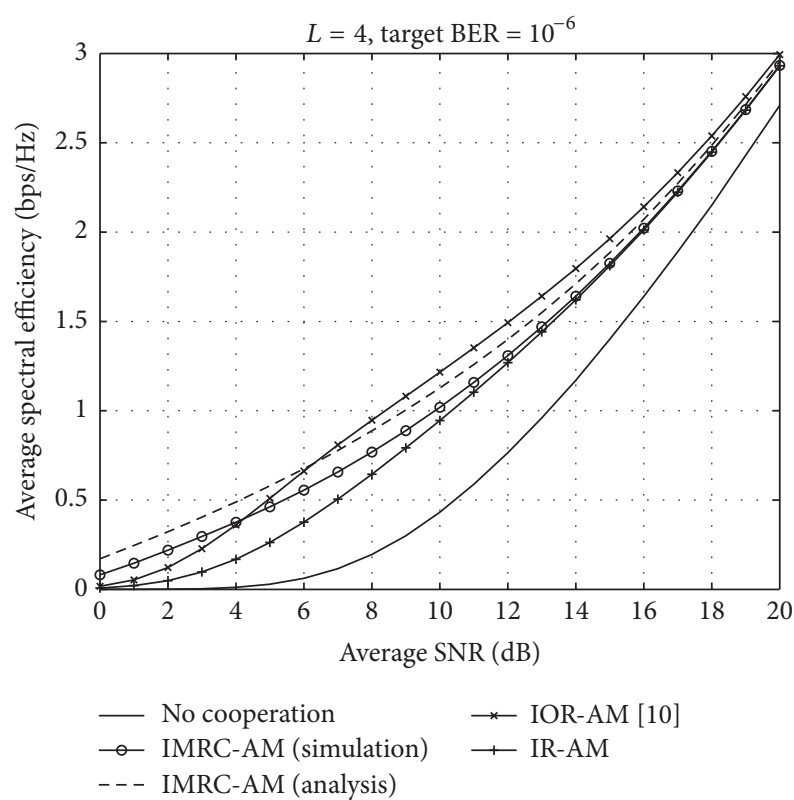

FIgURE 1: Comparison of the average spectral efficiency of the proposed IMRC-AM, IOR-AM, IR-AM, and noncooperation for a $10^{-6}$ BER performance over i.ni.d. Rayleigh fading channels.

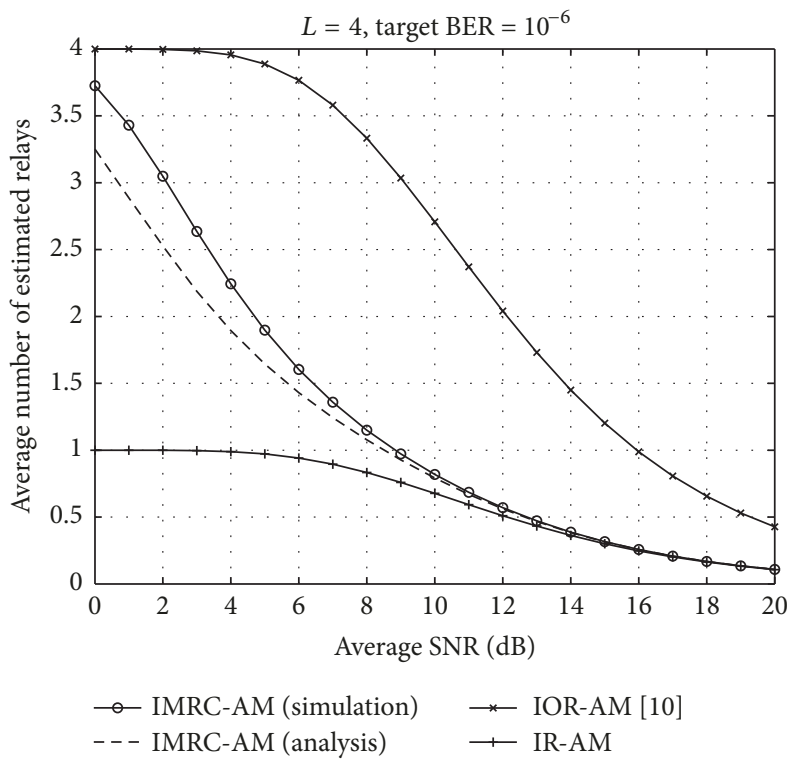

FIGURE 2: Comparison of the average number of estimated relays of the proposed IMRC-AM, IOR-AM, and IR-AM for a $10^{-6}$ BER performance over i.ni.d. Rayleigh fading channels.

orthogonal channels even if performance degradation may occur in the spectral efficiency. As shown in Figure 4, the provided lower bound analysis has also the same behavior as the simulation results.

In Figure 5, we show the effectiveness of the proposed IMRC-AM against the number of relay links with respect to the direct link. In order to give more useful insight, we constrain the total transmitted power to be the same irrespective of the number of relays, which implies the

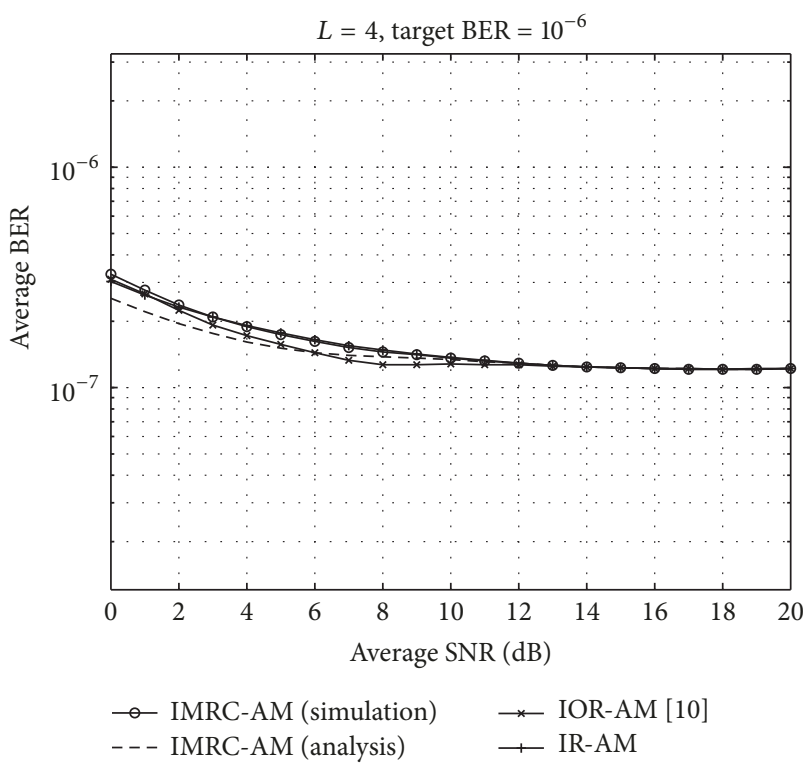

FIGURE 3: Average BER of the proposed scheme satisfying for a $10^{-6}$ BER required performance over i.ni.d. Rayleigh fading channels.

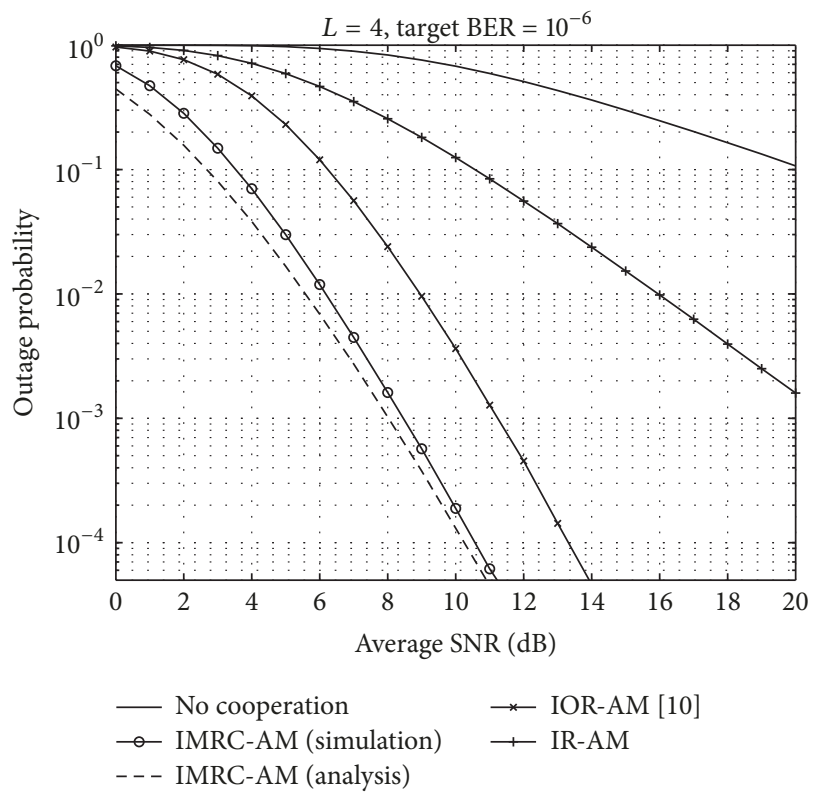

FIGURE 4: Outage probability of IMRC-AM, IOR-AM, IR-AM, and noncooperation for a $10^{-6}$ BER performance over i.ni.d. Rayleigh fading channels.

direct link fairly uses the total power against the number of relays. Since our proposed scheme adaptively decides the number of active relays in an incremental manner, we cannot decide the transmitted power before collecting all of channel information with the total power constraint. However, in this example, we assume that all of channel information are available to show a fair comparison with respect to the total power. In addition, our proposed scheme has some improvements in the average spectral efficiency compared to the direct transmission and the IR-AM and we can check the 


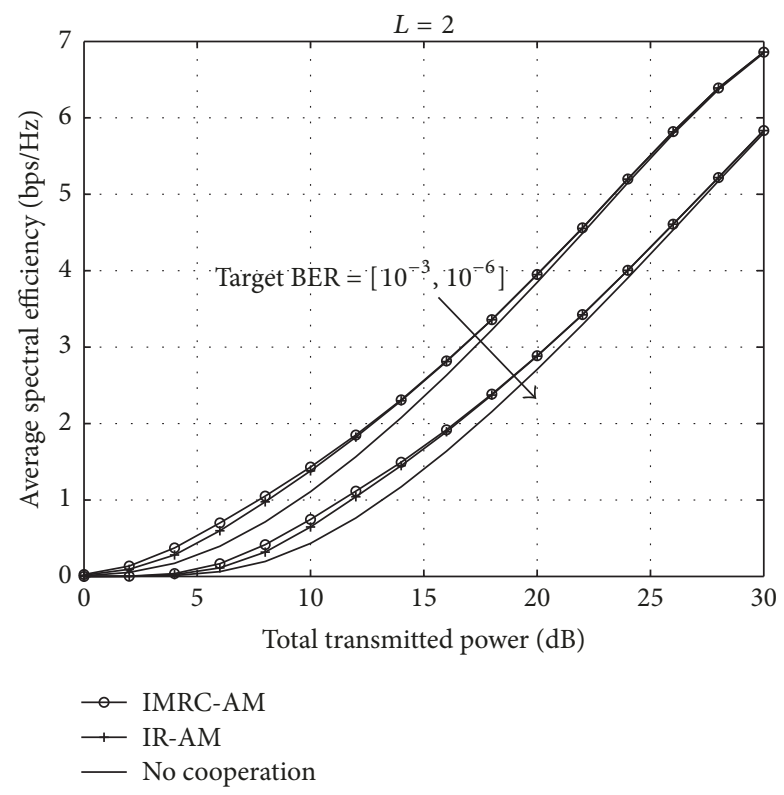

FIGURE 5: Comparison of the average spectral efficiency of IMRC$\mathrm{AM}$ with the different required BERs over i.ni.d. Rayleigh fading channels.

performance tradeoff between the spectral efficiency and the error performance for different target BERs with $L=2$ relays.

\section{Conclusion}

In this paper, we proposed IMRC-AM and analyzed its performance in terms of the average spectral efficiency, the average BER, outage probability, and the system complexity over the i.ni.d. Rayleigh fading channels. For a more tractable analysis, we used the upper bound for the output combined SNR and offered its statistical analyses such as CDF and PDF. From the selected numerical examples, we showed that our proposed scheme provided a certain improvement of the spectral efficiency and outage probability in comparison with the previous work while satisfying the required target BER performance. Finally, our provided performance bounds for IMRC-AM had a good agreement with the simulation results.

\section{Conflicts of Interest}

The authors declare that there are no conflicts of interest regarding the publication of this paper.

\section{Acknowledgments}

This research was supported by Basic Science Research Program through the National Research Foundation of Korea (NRF) funded by the Ministry of Science, ICT \& Future Planning under Grant 2015R1C1A1A02036516.

\section{References}

[1] J. N. Laneman, D. N. Tse, and G. W. Wornell, "Cooperative diversity in wireless networks: efficient protocols and outage behavior," Institute of Electrical and Electronics Engineers Transactions on Information Theory, vol. 50, no. 12, pp. 3062-3080, 2004.

[2] A. Bletsas, A. Khisti, D. P. Reed, and A. Lippman, "A simple cooperative diversity method based on network path selection," IEEE Journal on Selected Areas in Communications, vol. 24, no. 3, pp. 659-672, 2006.

[3] J. N. Laneman and G. W. Wornell, "Distributed space-time coded protocols for exploiting cooperative diversity in wireless networks," Institute of Electrical and Electronics Engineers Transactions on Information Theory, vol. 49, no. 10, pp. 2415-2425, 2003.

[4] Y. Jing and H. Jafarkhani, "Single and multiple relay selection schemes and their achievable diversity orders," IEEE Transactions on Wireless Communications, vol. 8, no. 3, pp. 1414-1423, 2009.

[5] J. Lee and N. Al-Dhahir, "Exploiting sparsity for multiple relay selection with relay gain control in large AF relay networks," IEEE Wireless Communications Letters, vol. 2, no. 3, pp. 347-350, 2013.

[6] G. Amarasuriya, M. Ardakani, and C. Tellambura, "Outputthreshold multiple-relay-selection scheme for cooperative wireless networks," IEEE Transactions on Vehicular Technology, vol. 59, no. 6, pp. 3091-3097, 2010.

[7] K.-S. Hwang and M. Ju, "Adaptive relay selection based on incremental relaying with output threshold," in Proceedings of the 2012 International Conference on ICT Convergence: "Global Open Innovation Summit for Smart ICT Convergence”, ICTC '12, pp. 39-43, IEEE, Jeju Island, Republic of Korea, October 2012.

[8] A. J. Goldsmith and S. G. Chua, "Variable-rate variable-power MQAM for fading channels," IEEE Transactions on Communications, vol. 45, no. 10, pp. 1218-1230, 1997.

[9] K.-S. Hwang, Y.-C. Ko, and M.-S. Alouini, "Performance analysis of incremental opportunistic relaying over identically and non-identically distributed cooperative paths," IEEE Transactions on Wireless Communications, vol. 8, no. 4, pp. 1953-1961, 2009.

[10] T. Nechiporenko, P. Kalansuriya, and C. Tellambura, "Performance of optimum switching adaptive M-QAM for amplifyand-forward relays," IEEE Transactions on Vehicular Technology, vol. 58, no. 5, pp. 2258-2268, 2009.

[11] M. Torabi, D. Haccoun, and J.-F. Frigon, "Relay selection in AF cooperative systems: an overview," IEEE Vehicular Technology Magazine, vol. 7, no. 4, pp. 104-113, 2012.

[12] B. Lee and C. Lee, "Performance analysis of incremental hop selection scheme with adaptive modulation for cooperative multi-hop networks," IEEE Transactions on Wireless Communications, vol. 14, no. 1, pp. 435-445, 2015.

[13] S. Sun, K. Yang, J. Wu, D. Zhu, and M. Lei, "Adaptive modulation and coding for two-way relaying with amplify-and-forward protocols," IET Communications, vol. 8, no. 7, pp. 1017-1023, 2014.

[14] H.-C. Yang and M.-S. Alouini, "MRC and GSC diversity combining with an output threshold," IEEE Transactions on Vehicular Technology, vol. 54, no. 3, pp. 1081-1090, 2005.

[15] P. A. Anghel and M. Kaveh, "Exact symbol error probability of a cooperative network in a rayleigh-fading environment," IEEE Transactions on Wireless Communications, vol. 3, no. 5, pp. 14161421, 2004.

[16] A. J. Goldsmith, Wireless Communications, Cambridge University Press, New York, NY, USA, 4th edition, 2005. 
[17] M. K. Simon and M.-S. Alouini, Digital Communication over Fading Channels, John Wiley and Sons, Inc., New York, NY, USA, 2nd edition, 2004.

[18] B. Choi and L. Hanzo, "Optimum mode-switching-assisted constant-power single- and multicarrier adaptive modulation," IEEE Transactions on Vehicular Technology, vol. 52, no. 3, pp. 536-560, 2003.

[19] T. S. Rappaport, Wireless Communications: Principles and Practice, Prentice Hall PTR, New Jersey, NJ, USA, 1996. 


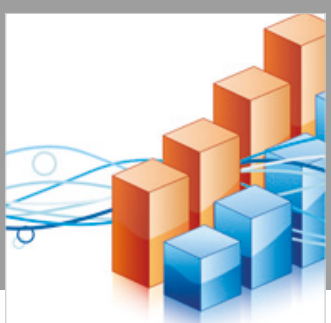

Advances in

Operations Research

\section{-n-m}
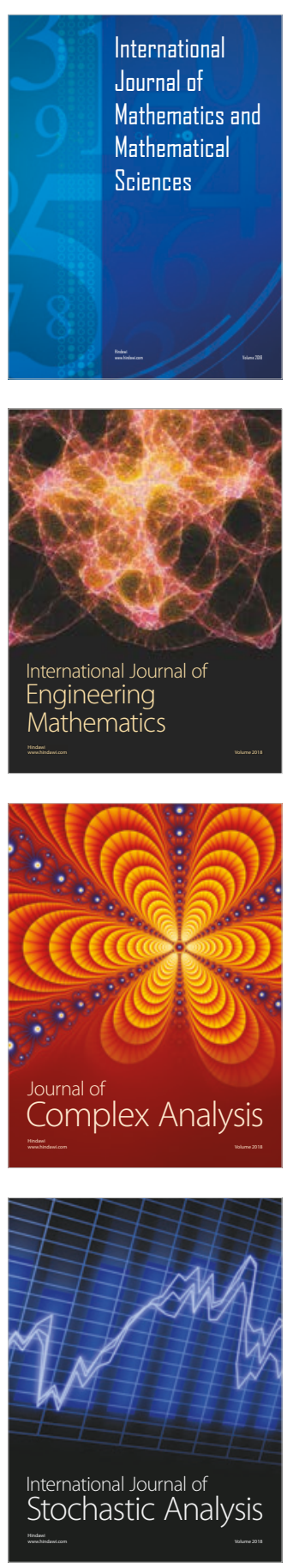
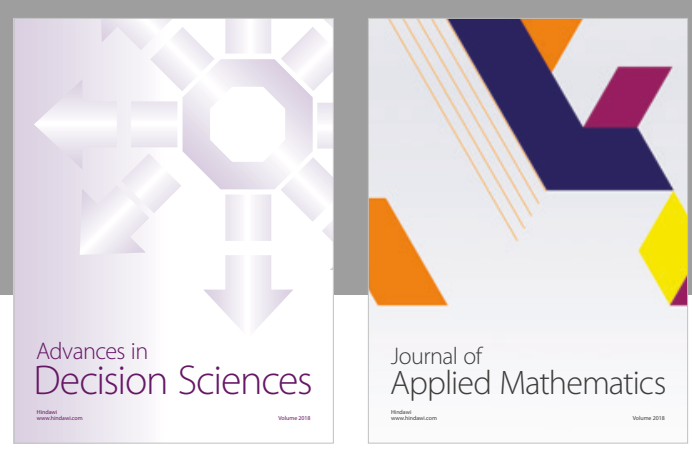

Journal of

Applied Mathematics
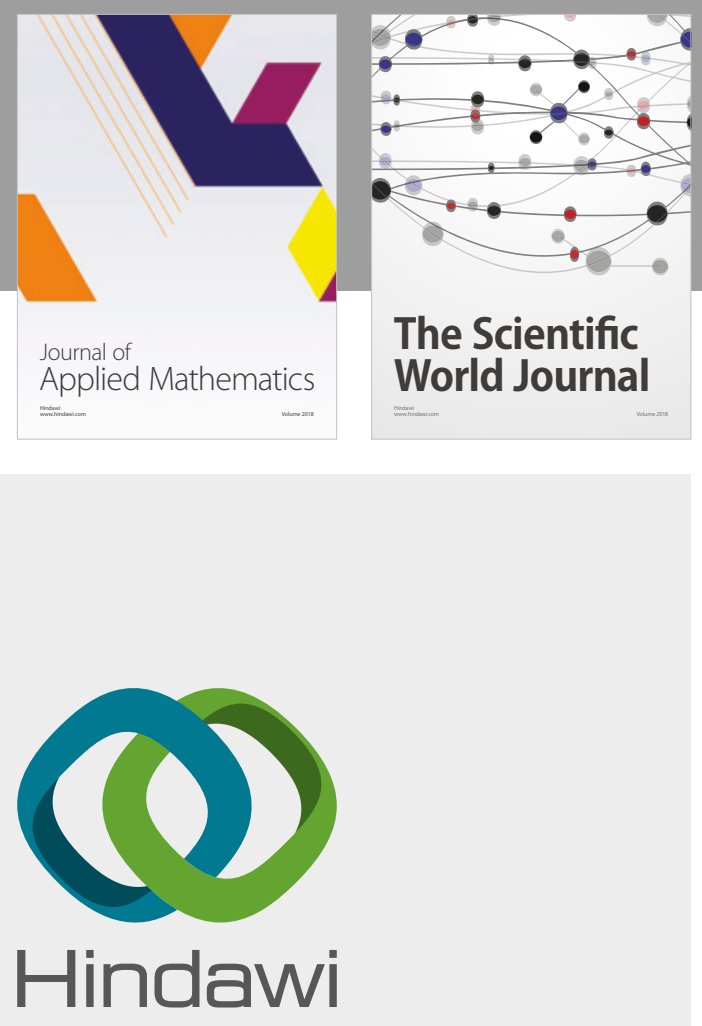

Submit your manuscripts at

www.hindawi.com

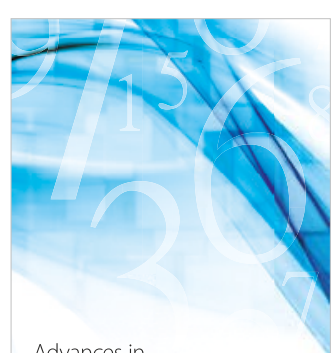

Advances in
Numerical Analysis
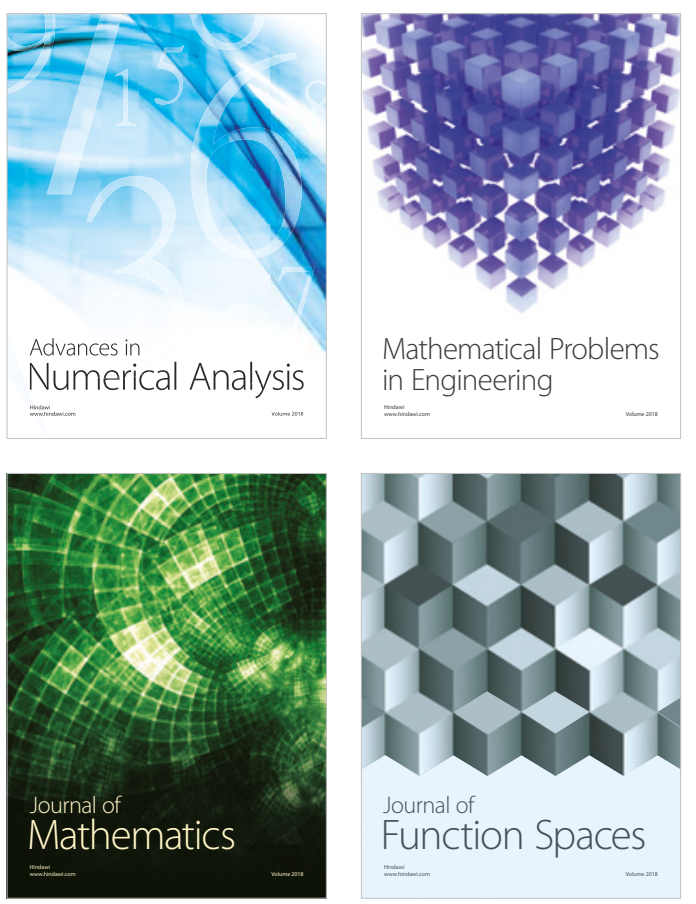

Mathematical Problems in Engineering

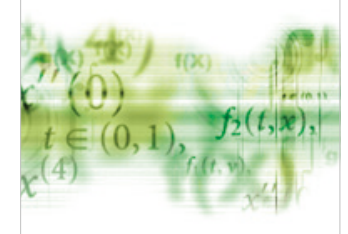

International Journal of

Differential Equations

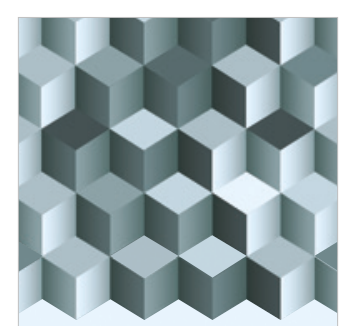

Journal of

Function Spaces

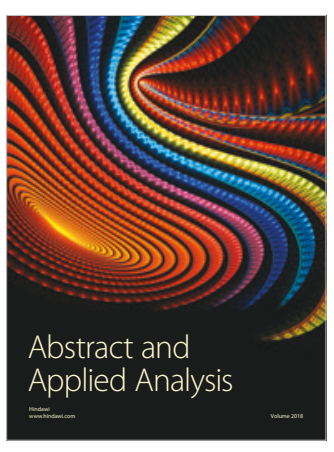

The Scientific

World Journal

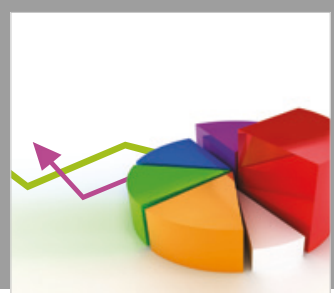

Journal of

Probability and Statistics
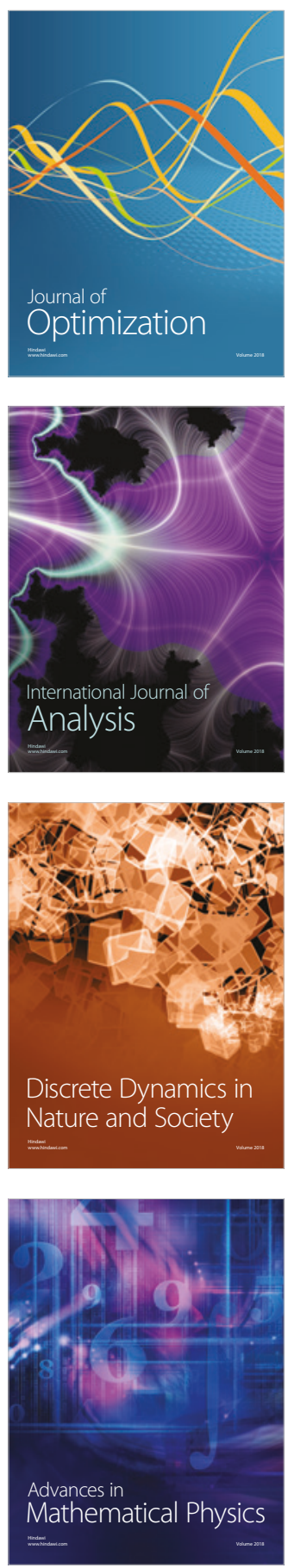